\title{
On Functions of Integrable Mean Oscillation
}

\author{
Oscar Blasco* and M. Amparo Pérez \\ Departamento de Análisis Matemático \\ Universidad de Valencia \\ 46100 Burjassot \\ Valencia - Spain \\ oblasco@uv.es
}

Recibido: 1 de diciembre de 2004

Aceptado: 21 de abril de 2005

\section{ABSTRACT}

Given $f \in L^{1}(\mathbb{T})$ we denote by $w_{\text {mo }}(f)$ the modulus of mean oscillation given by

$$
w_{\mathrm{mo}}(f)(t)=\sup _{0<|I| \leq t} \frac{1}{|I|} \int_{I}\left|f\left(e^{i \theta}\right)-m_{I}(f)\right| \frac{d \theta}{2 \pi}
$$

where $I$ is an arc of $\mathbb{T},|I|$ stands for the normalized length of $I$, and $m_{I}(f)=$ $\frac{1}{|I|} \int_{I} f\left(e^{i \theta}\right) \frac{d \theta}{2 \pi}$. Similarly we denote by $w_{\mathrm{ho}}(f)$ the modulus of harmonic oscillation given by

$$
w_{\mathrm{ho}}(f)(t)=\sup _{1-t \leq|z|<1} \int_{\mathbb{T}}\left|f\left(e^{i \theta}\right)-P(f)(z)\right| P_{z}\left(e^{i \theta}\right) \frac{d \theta}{2 \pi}
$$

where $P_{z}\left(e^{i \theta}\right)$ and $P(f)$ stand for the Poisson kernel and the Poisson integral of $f$ respectively.

It is shown that, for each $0<p<\infty$, there exists $C_{p}>0$ such that

$$
\int_{0}^{1}\left[w_{\mathrm{mo}}(f)(t)\right]^{p} \frac{d t}{t} \leq \int_{0}^{1}\left[w_{\mathrm{ho}}(f)(t)\right]^{p} \frac{d t}{t} \leq C_{p} \int_{0}^{1}\left[w_{\mathrm{mo}}(f)(t)\right]^{p} \frac{d t}{t} .
$$

Key words: mean oscillation, BMO, modulus of continuity.

2000 Mathematics Subject Classification: 46B25.

*Partially supported by Proyecto BMF2002-0401 and MTN2004-21420-E 


\section{Introduction.}

As usual we denote by BMO the space of functions $f \in L^{1}(\mathbb{T})$ such that

$$
\|f\|_{*}=\sup _{I \subseteq \mathbb{T}} \frac{1}{|I|} \int_{I}\left|f\left(e^{i \theta}\right)-m_{I}(f)\right| \frac{d \theta}{2 \pi}<\infty
$$

where $I$ is an arc of the circle $\mathbb{T},|I|$ stands for the normalized length of $I$ and $m_{I}(f)=\frac{1}{|I|} \int_{I} f\left(e^{i \theta}\right) \frac{d \theta}{2 \pi}$. We write $\|f\|_{\text {BMO }}=|\hat{f}(0)|+\|f\|_{*}$.

If $f \in L^{1}(\mathbb{T})$ and $0<t \leq 1$, we define the modulus of mean oscillation of $f$ at the point $t$ as

$$
w_{\mathrm{mo}}(f)(t)=\sup _{0<|I| \leq t} \frac{1}{|I|} \int_{I}\left|f\left(e^{i \theta}\right)-m_{I}(f)\right| \frac{d \theta}{2 \pi} .
$$

Clearly, for $0<t \leq s<1$, one has

$$
\sup _{t<|I| \leq s} \frac{1}{|I|} \int_{I}\left|f\left(e^{i \theta}\right)-m_{I}(f)\right| \frac{d \theta}{2 \pi} \leq \frac{2}{t}\|f\|_{1} .
$$

Hence, for $0<t \leq s<1$, one has

$$
w_{\mathrm{mo}}(f)(t) \leq w_{\mathrm{mo}}(f)(s) \leq \max \left\{w_{\mathrm{mo}}(f)(t), \frac{2\|f\|_{1}}{t}\right\} .
$$

In particular, $f \in \mathrm{BMO}$ if and only if $w_{\mathrm{mo}}(f)(t)<\infty$ for some (or for all) $0<t \leq 1$.

It is known that one can consider other equivalent moduli to define BMO. For instance, for $0<q<\infty$,

$$
w_{\mathrm{mo}, q}(f)(t)=\sup _{0<|I| \leq t}\left(\frac{1}{|I|} \int_{I}\left|f\left(e^{i \theta}\right)-m_{I}(f)\right|^{q} \frac{d \theta}{2 \pi}\right)^{1 / q} .
$$

It is also well known, by the John-Nirenberg lemma (see $[7,8]$ ), that there exist $C_{1}, C_{2}>0$ such that for all $\lambda>0$ and any arc $I$ with $|I| \leq t$,

$$
\frac{\left|\left\{\theta \in \mathbb{T}:\left|f\left(e^{i \theta}\right)-m_{I}\right|>\lambda\right\}\right|}{|I|} \leq C_{1} e^{-\frac{C_{2} \lambda}{w_{\operatorname{mo}}(f)(t)}}
$$

From here one gets that, for all $t>0$,

$$
w_{\mathrm{mo}}(f)(t) \approx w_{\mathrm{mo}, q}(f)(t) .
$$

One can also consider

$$
w_{\mathrm{mo}}^{\prime}(f)(t)=\sup _{|I| \leq t}\left(\frac{1}{|I|^{2}} \int_{I} \int_{I}\left|f\left(e^{i \theta}\right)-f\left(e^{i \varphi}\right)\right| \frac{d \theta}{2 \pi} \frac{d \varphi}{2 \pi}\right)
$$


or

$$
\tilde{w}_{\mathrm{mo}}(f)(t)=\sup _{|I| \leq t}\left(\inf _{c}\left(\frac{1}{|I|} \int_{I}\left|f\left(e^{i \theta}\right)-c\right| \frac{d \theta}{2 \pi}\right)\right)
$$

Clearly one gets

$$
w_{\mathrm{mo}}(f)(t) \leq w_{\mathrm{mo}}^{\prime}(f)(t) \leq 2 w_{\mathrm{mo}}(f)(t)
$$

and

$$
\tilde{w}_{\mathrm{mo}}(f)(t) \leq w_{\mathrm{mo}}(f)(t) \leq 2 \tilde{w}_{\mathrm{mo}}(f)(t) .
$$

A function $f$ is said to have vanishing mean oscillation, in short $f \in \mathrm{VMO}$, if

$$
\lim _{|I| \rightarrow 0} \frac{1}{|I|} \int_{I}\left|f\left(e^{i \theta}\right)-m_{I}(f)\right| \frac{d \theta}{2 \pi}=0 .
$$

This is a closed subspace of BMO, which can be characterized in many ways (see $[7,8,15]$ ).

Theorem 1.1. Let $f \in \mathrm{BMO}$. The following statements are equivalent:

(i) $f \in \mathrm{VMO}$.

(ii) $\lim _{t \rightarrow 0^{+}}\left\|T_{t} f-f\right\|_{\mathrm{BMO}}=0$, where $T_{t} f\left(e^{i \theta}\right)=f\left(e^{i(\theta-t)}\right)$.

(iii) $\lim _{r \rightarrow 1}\left\|P_{r} * f-f\right\|_{\mathrm{BMO}}=0$, where $P_{r}\left(e^{i \theta}\right)=\Re\left(\frac{1+r e^{-i \theta}}{1-r e^{-i \theta}}\right)$.

(iv) $f$ belongs to the closure of $C(\mathbb{T})$ in BMO.

(v) $\lim _{t \rightarrow 0^{+}} w_{\mathrm{mo}}(f)(t)=0$.

A generalization of $\mathrm{BMO}$ is the space $\operatorname{BMO}(\rho)$, consisting of functions $f \in L^{1}(\mathbb{T})$ such that $w_{\text {mo }}(f)(t)=O(\rho(t))$ for a fixed function $\rho$ with certain properties. The space $\operatorname{BMO}(\rho)$ has been considered by various authors (see $[10,15,17]$ ).

Our aim will be to analyze spaces where the function $\rho$ is not explicitly given, but we do know its behavior at the origin in terms of certain integrability conditions.

Given $0<p<\infty$, we shall denote by $\mathrm{MO}^{p}(\mathbb{T})$ the space of integrable functions such that $\int_{0}^{1}\left[w_{\text {mo }}(f)(t)\right]^{p} \frac{d t}{t}<\infty$.

Due to (2), the spaces $\mathrm{MO}_{q}^{p}$ of functions such that $\int_{0}^{1}\left[w_{\mathrm{mo}, q}(f)(t)\right]^{p} \frac{d t}{t}<\infty$ are all the same for $0<q<\infty$.

These spaces were considered in [12] (see page 74), under a different notation. Also some spaces $\mathrm{MO}_{s, r}^{\alpha}$, which are closely related to the ones considered in this paper, were introduced in [13].

We use the notations

$$
\omega_{\infty}(f)(t)=\sup _{|\theta-\varphi| \leq t}\left|f\left(e^{i \theta}\right)-f\left(e^{i \varphi}\right)\right|
$$


and

$$
\omega_{q}(f)(t)=\sup _{|u| \leq t}\left(\int_{\mathbb{T}}\left|f\left(e^{i(\theta+u)}\right)-f\left(e^{i \theta}\right)\right|^{q} \frac{d \theta}{2 \pi}\right)^{1 / q}
$$

for $0<q<\infty$.

Now, for $0<s<1$ and $0<p, q \leq \infty$, the Besov space $B_{q, p}^{s}(\mathbb{T})$ consists of functions in $L^{q}(\mathbb{T})$ such that $t^{-s} \omega_{q}(f) \in L^{p}\left((0,2 \pi), \frac{d t}{t}\right)$. Of course the cases $B_{q, \infty}^{s}$ where $0<q \leq \infty$ correspond to Lipschitz or Hölder classes, to be denoted $\operatorname{Lip}_{s}(\mathbb{T})$ instead of $B_{\infty, \infty}^{s}(\mathbb{T})$.

We denote by $X_{p}$ the space consisting of functions $L^{\infty}(\mathbb{T})$ such that $\omega_{\infty}(f) \in$ $L^{p}\left((0,2 \pi), \frac{d t}{t}\right)$.

From (3) we easily obtain, for any $t>0$,

$$
w_{\mathrm{mo}}(f)(t) \leq C \omega_{\infty}(f)(t) .
$$

Hence $X_{p} \subset M O^{p}(\mathbb{T})$ for any $0<p<\infty$.

On the other hand, if $I, J$ are $\operatorname{arcs}$ on $\mathbb{T}$ such that $I \subset J$ then

$$
\left|m_{J}(f)-m_{I}(f)\right| \leq \frac{|J|}{|I|} w_{\mathrm{mo}}(f)(|J|) .
$$

Now, given $I$ with $|I| \leq t$, using the Lebesgue differentiation theorem, one gets

$$
f\left(e^{i \theta}\right)=\lim _{n} m_{I_{n}} f, \quad \text { for a.a. } \theta \in I,
$$

where $I_{n}$ is a decreasing sequence of $\operatorname{arcs}$ containing $\theta$ such that $\left|I_{n}\right|=2\left|I_{n+1}\right|$.

Hence using (4), we have that for any $f \in \mathrm{MO}^{1}(\mathbb{T})$

$$
\begin{aligned}
\left|f\left(e^{i \theta}\right)-m_{I}\right| & \leq \lim _{n}\left|m_{I_{n}} f-m_{I} f\right| \\
& \leq \sum_{k=1}^{\infty}\left|m_{I_{k}} f-m_{I_{k-1}} f\right| \\
& \leq C \sum_{k=1}^{\infty} w_{\mathrm{mo}}(f)\left(2^{-k} t\right) \\
& \leq C \int_{0}^{t} w_{\mathrm{mo}}(f)(s) \frac{d s}{s} .
\end{aligned}
$$

Therefore we obtain, for any $t>0$,

$$
\omega_{\infty}(f)(t) \leq 2 C \int_{0}^{t} w_{\mathrm{mo}}(f)(s) \frac{d s}{s} .
$$

This implies that $\mathrm{MO}^{1}(\mathbb{T}) \subset \operatorname{Lip}_{\phi}$, where $\operatorname{Lip}_{\phi}$ stands for the space of continuous functions such that

$$
\left|f\left(e^{i \theta}\right)-f\left(e^{i \varphi}\right)\right| \leq C \phi(|\theta-\varphi|),
$$


for $\phi(t)=\sup \left\{\int_{0}^{t} w_{\operatorname{mo}}(f)(s) \frac{d s}{s}: \int_{0}^{1} w_{\operatorname{mo}}(f)(t) \frac{d t}{t} \leq 1\right\}$.

BMO-type characterizations of these spaces have been extensively considered in the literature. The reader is referred to $[2,3,9]$ for the case $p=\infty$ and to $[4,5]$ for the cases $0<s$ and $1<p<\infty$.

We shall consider a description of $\mathrm{MO}^{p}(\mathbb{T})$ where the averages over arcs are replaced by averages with respect the Poisson kernel.

We denote by $\mathrm{BMOH}$ the space of functions $f \in L^{1}(\mathbb{T})$ such that

$$
\|f\|_{* *}=\sup _{z \in \Delta} \int_{\mathbb{T}}\left|f\left(e^{i \theta}\right)-P(f)(z)\right| P_{z}\left(e^{i \theta}\right) \frac{d \theta}{2 \pi}<\infty,
$$

where $\Delta$ denotes the open unit disc, $P(f)(z)=\int_{\mathbb{T}} f\left(e^{i \theta}\right) P_{z}\left(e^{i \theta}\right) \frac{d \theta}{2 \pi}$ and $P_{z}\left(e^{i \theta}\right)=$ $\Re\left(\frac{1+z e^{-i \theta}}{1-z e^{-i \theta}}\right)$. We write $\|f\|_{\text {BMOH }}=|P(f)(0)|+\|f\|_{* *}$.

It is not difficult to prove (see $[7,8]$ ) that $f \in \mathrm{BMO}$ if and only if $f \in \mathrm{BMOH}$ with equivalent norms.

In this situation we define the modulus of harmonic oscillation of $f$ at the point $t$ as

$$
w_{\text {ho }}(f)(t)=\sup _{1-t \leq|z|<1} \int_{\mathbb{T}}\left|f\left(e^{i \theta}\right)-P(f)(z)\right| P_{z}\left(e^{i \theta}\right) \frac{d \theta}{2 \pi} .
$$

Hence, $f \in$ BMO (respect. BMOH) if and only if $w_{\text {mo }}(f)(1)<\infty$ (respect. $w_{\text {ho }}(f)(1)$ $<\infty)$.

For $0<p<\infty$, we denote by $\mathrm{HO}^{p}(\mathbb{T})$ the space of $f \in L^{1}(\mathbb{T})$ such that $\int_{0}^{1}\left[w_{\mathrm{ho}}(f)(t)\right]^{p} \frac{d t}{t}<\infty$.

Of course one can also use other moduli to define this space. For instance, for $0<q<\infty$,

$$
w_{\mathrm{ho}, q}(f)(t)=\sup _{1-t \leq|z|<1}\left(\int_{\mathbb{T}}\left|f\left(e^{i \theta}\right)-P(f)(z)\right|^{q} P_{z}\left(e^{i \theta}\right) \frac{d \theta}{2 \pi}\right)^{1 / q},
$$

or

$$
\tilde{w}_{\text {ho }}(f)(t)=\sup _{1-t \leq|z|<1} \inf _{c} \int_{\mathbb{T}}\left|f\left(e^{i \theta}\right)-c\right| P_{z}\left(e^{i \theta}\right) \frac{d \theta}{2 \pi} .
$$

The main objective of the paper is to show that, for $0<p<\infty$, we have $\mathrm{MO}^{p}(\mathbb{T})=$ $\mathrm{HO}^{p}(\mathbb{T})$ and with equivalent "norms".

The paper is divided into two sections. The first one is devoted to introducing $\mathrm{MO}^{p}(\mathbb{T})$ and proving some of its properties and the second one to introducing $\mathrm{HO}^{p}(\mathbb{T})$ and to showing that $\mathrm{HO}^{p}(\mathbb{T})$ coincides with $\mathrm{MO}^{p}(\mathbb{T})$.

\section{Integrable mean oscillation.}

We will see first that the modulus of mean oscillation is continuous. We shall use the following lemma. 
Lemma 2.1. Let $f \in L^{1}(\mathbb{T})$. If $\left\{I_{n}\right\}$ is a sequence of arcs such that $\lim _{n \rightarrow \infty} I_{n}=I$ for some arc $I$ with $|I|>0$ then

$$
\lim _{n \rightarrow \infty} \frac{1}{\left|I_{n}\right|} \int_{I_{n}}\left|f\left(e^{i \theta}\right)-m_{I_{n}}(f)\right| \frac{d \theta}{2 \pi}=\frac{1}{|I|} \int_{I}\left|f\left(e^{i \theta}\right)-m_{I}(f)\right| \frac{d \theta}{2 \pi} .
$$

Proof. Let us first estimate

$$
\begin{aligned}
\frac{1}{\left|I_{n}\right|} \int_{I_{n}}\left|f\left(e^{i \theta}\right)-m_{I_{n}}(f)\right| & \frac{d \theta}{2 \pi}-\frac{1}{|I|} \int_{I}\left|f\left(e^{i \theta}\right)-m_{I}(f)\right| \frac{d \theta}{2 \pi} \\
\leq & \frac{1}{\left|I_{n}\right|} \int_{I_{n}}\left|f\left(e^{i \theta}\right)-m_{I}(f)\right| \frac{d \theta}{2 \pi}+\left|m_{I_{n}}(f)-m_{I}(f)\right| \\
& -\frac{1}{|I|} \int_{I}\left|f\left(e^{i \theta}\right)-m_{I}(f)\right| \frac{d \theta}{2 \pi} \\
\leq & \frac{1}{|I|}\left(\int_{I_{n}}\left|f\left(e^{i \theta}\right)-m_{I}(f)\right| \frac{d \theta}{2 \pi}-\int_{I}\left|f\left(e^{i \theta}\right)-m_{I}(f)\right| \frac{d \theta}{2 \pi}\right) \\
& +\left|m_{I_{n}}(f)-m_{I}(f)\right|+2|| f \|_{1}\left(\frac{1}{\left|I_{n}\right|}-\frac{1}{|I|}\right) .
\end{aligned}
$$

Notice that $\nu(A)=\int_{A} f\left(e^{i \theta}\right) \frac{d \theta}{2 \pi}$ and $\mu^{I}(A)=\int_{A}\left|f\left(e^{i \theta}\right)-m_{I}(f)\right| \frac{d \theta}{2 \pi}$ are a complex and a positive measure respectively with integrable densities. Therefore the result follows passing to the limit as $n$ goes to $\infty$.

Proposition 2.2. Let $f \in \mathrm{BMO}$. Then $w_{\mathrm{mo}}(f)$ is increasing and continuous in $(0,1]$.

Proof. Monotonicity has already been proved in our formula (1).

Let $0<t_{0} \leq 1$ and let us prove that it is left continuous at $t_{0}$. Given $\varepsilon>0$ we find $I_{t_{0}} \subset \mathbb{T}$ such that $0<\left|I_{t_{0}}\right| \leq t_{0}$ and

$$
w_{\mathrm{mo}}(f)\left(t_{0}\right) \leq \frac{1}{\left|I_{t_{0}}\right|} \int_{I_{t_{0}}}\left|f\left(e^{i \theta}\right)-m_{I_{t_{0}}}(f)\right| \frac{d \theta}{2 \pi}+\frac{\varepsilon}{2} .
$$

Let $\left(t_{n}\right)$ be a sequence such that $t_{n} \leq t_{0}$ for all $n \in \mathbb{N}$ and converges to $t_{0}$.

If $\left|I_{t_{0}}\right|=t_{0}$, we can find $I_{n} \subset I_{t_{0}}$ such that $\lim _{n \rightarrow \infty} I_{n}=I_{t_{0}}$. Hence

$$
\begin{aligned}
w_{\mathrm{mo}}(f)\left(t_{0}\right) & -w_{\mathrm{mo}}(f)\left(t_{n}\right) \leq \\
& \leq \frac{1}{\left|I_{t_{0}}\right|} \int_{I_{t_{0}}}\left|f\left(e^{i \theta}\right)-m_{I_{t_{0}}}(f)\right| \frac{d \theta}{2 \pi}-\frac{1}{\left|I_{n}\right|} \int_{I_{n}}\left|f\left(e^{i \theta}\right)-m_{I_{n}}(f)\right| \frac{d \theta}{2 \pi}+\frac{\varepsilon}{2} .
\end{aligned}
$$

Now use Lemma 2.1 to get $\lim _{n \rightarrow \infty} w_{\mathrm{mo}}(f)\left(t_{0}\right)-w_{\mathrm{mo}}(f)\left(t_{n}\right)=0$.

If $\left|I_{t_{0}}\right|<t_{0}$ there exists $n_{0}$ such that $\left|I_{t_{0}}\right| \leq t_{n}$ for $n \geq n_{0}$. Hence $w_{\text {mo }}(f)\left(t_{0}\right)-$ $w_{\mathrm{mo}}(f)\left(t_{n}\right)<\frac{\varepsilon}{2}$ for $n \geq n_{0}$. 
To see that it is right continuous at $t_{0}$, we shall argue as follows: Let $\left(t_{n}\right)$ be a sequence such that $t_{n} \geq t_{0}$ for all $n \in \mathbb{N}$ and converges to $t_{0}$. We shall find a subsequence $\left(t_{n_{k}}\right)$ such that $\lim _{k \rightarrow \infty} w_{\text {mo }}(f)\left(t_{n_{k}}\right)=w_{\text {mo }}(f)\left(t_{0}\right)$.

Given $\varepsilon>0$ we find $I_{n} \subset \mathbb{T}$ such that $0<\left|I_{n}\right| \leq t_{n}$ and

$$
w_{\mathrm{mo}}(f)\left(t_{n}\right) \leq \frac{1}{\left|I_{n}\right|} \int_{I_{n}}\left|f\left(e^{i \theta}\right)-m_{I_{n}}(f)\right| \frac{d \theta}{2 \pi}+\varepsilon
$$

Let $\mathcal{F}=\left\{n \in \mathbb{N}:\left|I_{n}\right|>t_{0}\right\}$. If $\mathcal{F}$ is finite then $\left|I_{n}\right| \leq t_{0}$ for $n \geq n_{0}$ and

$$
w_{\mathrm{mo}}(f)\left(t_{n}\right)-w_{\mathrm{mo}}(f)\left(t_{0}\right)<\varepsilon \quad \text { for } n \geq n_{0} .
$$

Without loss of generality we assume $\left|I_{n}\right|>t_{0}$ for all $n \in \mathbb{N}$.

Call $I_{0}=\cup_{n=1}^{\infty} \cap_{k=n}^{\infty} I_{k}$. It is easy to see that $I_{0}$ is an arc and that $\left|I_{0}\right|=t_{0}$. Take a subsequence $n_{k}$ such that $\left(I_{n_{k}}\right)$ converges to $I_{0}$. We have

$$
\begin{aligned}
w_{\mathrm{mo}}(f)\left(t_{n_{k}}\right) & -w_{\mathrm{mo}}(f)\left(t_{0}\right) \leq w_{\mathrm{mo}}(f)\left(t_{n_{k}}\right)-\frac{1}{\left|I_{0}\right|} \int_{I_{0}}\left|f\left(e^{i \theta}\right)-m_{I_{0}}(f)\right| \frac{d \theta}{2 \pi} \\
& \leq \frac{1}{\left|I_{n_{k}}\right|} \int_{I_{n_{k}}}\left|f\left(e^{i \theta}\right)-m_{I_{n_{k}}}(f)\right| \frac{d \theta}{2 \pi}-\frac{1}{\left|I_{0}\right|} \int_{I_{0}}\left|f\left(e^{i \theta}\right)-m_{I_{0}}(f)\right| \frac{d \theta}{2 \pi}+\varepsilon .
\end{aligned}
$$

The proof is complete invoking Lemma 2.1.

Remark 2.3. Let $f \in \mathrm{BMO}$ and take $a(f)=\lim _{t \rightarrow 0^{+}} w_{\mathrm{mo}}(f)(t)$. Hence $f \in \mathrm{VMO}$ if and only if $a(f)=0$.

For each $0<p<\infty$, we define the quasi-norm (norm for $p \geq 1$ ) on $\mathrm{MO}^{p}(\mathbb{T})$ by

$$
\|f\|_{\mathrm{MO}^{p}}=\|f\|_{L^{1}(\mathbb{T})}+\left(\int_{0}^{1}\left[w_{\mathrm{mo}}(f)(t)\right]^{p} \frac{d t}{t}\right)^{1 / p} .
$$

Although the next result is probably known, we include a proof for the sake of completeness.

Theorem 2.4. Let $0<p<\infty$. Then $\left(\mathrm{MO}^{p}(\mathbb{T}),\|\cdot\|_{\mathrm{MO}^{p}}\right)$ is a complete space.

Proof. Let $\left\{f_{n}\right\}$ be a Cauchy sequence in $\mathrm{MO}^{p}(\mathbb{T})$. In particular, there exists $f \in \mathrm{BMO}$ such that $\left\{f_{n}\right\}$ converges to $f$.

Let $|I| \leq t, 0<t \leq 1$. Using that $f_{n} \rightarrow f$ in $L^{1}(\mathbb{T})$ we get that $m_{I}\left(f_{n}\right) \rightarrow m_{I}(f)$ and that there exists a subsequence $\left(n_{k}\right)$, such that $f_{n_{k}} \rightarrow f$ a.e. 
Now

$$
\begin{aligned}
\frac{1}{|I|} \int_{I} \mid f_{n}\left(e^{i \theta}\right)-f\left(e^{i \theta}\right)- & m_{I}\left(f_{n}-f\right) \mid \frac{d \theta}{2 \pi} \\
& =\frac{1}{|I|} \int_{I}\left|f_{n}\left(e^{i \theta}\right)-\lim _{k} f_{n_{k}}\left(e^{i \theta}\right)-\lim _{k} m_{I}\left(f_{n}-f_{n_{k}}\right)\right| \frac{d \theta}{2 \pi} \\
& =\frac{1}{|I|} \int_{I} \lim _{k}\left|f_{n}\left(e^{i \theta}\right)-f_{n_{k}}\left(e^{i \theta}\right)-m_{I}\left(f_{n}-f_{n_{k}}\right)\right| \frac{d \theta}{2 \pi} \\
& \leq \liminf _{k} \frac{1}{|I|} \int_{I}\left|f_{n}\left(e^{i \theta}\right)-f_{n_{k}}\left(e^{i \theta}\right)-m_{I}\left(f_{n}-f_{n_{k}}\right)\right| \frac{d \theta}{2 \pi} \\
& \leq \liminf _{k} w_{\mathrm{mo}}\left(f_{n}-f_{n_{k}}\right)(t) .
\end{aligned}
$$

Therefore

$$
w_{\mathrm{mo}}\left(f_{n}-f\right)(t) \leq \liminf _{k} w_{\mathrm{mo}}\left(f_{n}-f_{n_{k}}\right)(t)
$$

Hence

$$
\begin{aligned}
\int_{0}^{1}\left[w_{\mathrm{mo}}\left(f_{n}-f\right)(t) \frac{d t}{t}\right]^{p} & \leq \int_{0}^{1} \liminf _{k}\left[w_{\mathrm{mo}}\left(f_{n}-f_{n_{k}}\right)(t)\right]^{p} \frac{d t}{t} \\
& \leq \liminf _{k} \int_{0}^{1}\left[w_{\mathrm{mo}}\left(f_{n}-f_{n_{k}}\right)(t)\right]^{p} \frac{d t}{t}
\end{aligned}
$$

Finally, using that $f_{n}$ is a Cauchy sequence we get $\lim _{n \rightarrow \infty}\left\|f_{n}-f\right\|_{\mathrm{MO}^{p}}=0$ and that $f \in \mathrm{MO}^{p}$.

Proposition 2.5. Let $0<p \leq q<\infty$ and $s>0$.

(i) $\mathrm{MO}^{p}(\mathbb{T}) \subseteq \mathrm{MO}^{q}(\mathbb{T})$.

(ii) $\operatorname{Lip}_{s}(\mathbb{T}) \subset \bigcap_{p>0} \mathrm{MO}^{p}(\mathbb{T}) \subset \mathrm{MO}^{1}(\mathbb{T}) \subset C(\mathbb{T})$.

(iii) $\bigcup_{p>0} \mathrm{MO}^{p}(\mathbb{T}) \subset \mathrm{VMO}$.

Proof. (i) It is a consequence of the following fact:

$$
\left(\int_{0}^{1}\left[w_{\mathrm{mo}}(f)(t)\right]^{p} \frac{d t}{t}\right)^{1 / p} \approx\left(\sum_{k=0}^{\infty}\left[w_{\mathrm{mo}}(f)\left(2^{-k}\right)\right]^{p}\right)^{1 / p} .
$$

(ii) Note that $f \in \operatorname{Lip}_{s}$ if and only if $w_{\mathrm{mo}}(f)(t) \leq C t^{s}$. This gives the first inclusion.

The fact that $\mathrm{MO}^{1}(\mathbb{T}) \subset C(\mathbb{T})$ follows by $(5)$.

(iii) Observe that, for any $p>0$ and $t>0$, one has

$$
w_{\mathrm{mo}}(f)^{p}(t) \log \frac{1}{t} \leq \int_{t}^{1} w_{\mathrm{mo}}(f)^{p}(u) \frac{d u}{u} \leq\|f\|_{\mathrm{MO}^{p}} .
$$

Hence $\lim _{t \rightarrow 0^{+}} w_{\mathrm{mo}}(f)(t)=0$ for $f \in \cup_{p>0} \mathrm{MO}^{p}(\mathbb{T})$. 
Let us point out some properties of BMO that are shared by these spaces.

Proposition 2.6. If $f \in \mathrm{MO}^{p}(\mathbb{T})$ then $|f| \in \mathrm{MO}^{p}(\mathbb{T})$.

Proof. Let $t \in(0,1)$ and $I \subset \mathbb{T}$ with $|I| \leq t$. Then

$$
\begin{aligned}
\frac{1}{|I|} \int_{I}|| f\left(e^{i \theta}\right)\left|-m_{I}(|f|)\right| \frac{d \theta}{2 \pi} & \leq \frac{1}{|I|} \int_{I}|| f\left(e^{i \theta}\right)|-| m_{I}(f)|| \frac{d \theta}{2 \pi} \\
& +\left|m_{I}(|f|)-\right| m_{I}(f)|| \\
& \leq \frac{2}{|I|} \int_{I}|| f\left(e^{i \theta}\right)|-| m_{I}(f)|| \frac{d \theta}{2 \pi} \\
& \leq \frac{2}{|I|} \int_{I}\left|f\left(e^{i \theta}\right)-m_{I}(f)\right| \frac{d \theta}{2 \pi}
\end{aligned}
$$

This shows that $w_{\mathrm{mo}}(|f|)(t) \leq 2 w_{\mathrm{mo}}(f)(t)$ and the proof is complete.

Recall that $T_{t}$ denotes the translation operator, that is $T_{t} f\left(e^{i \theta}\right)=f\left(e^{i(\theta-t)}\right)$. We have the following result.

Theorem 2.7. Let $0<p<\infty$ and $f \in \mathrm{MO}^{p}(\mathbb{T})$. Then

$$
\lim _{s \rightarrow 0^{+}}\left\|T_{s} f-f\right\|_{\mathrm{MO}^{p}}=0 .
$$

Proof. Due to (iii) in Proposition $2.5 f \in$ VMO. Now Theorem 1.1 gives that $\lim _{s \rightarrow 0^{+}}\left\|T_{s} f-f\right\|_{\mathrm{BMO}}=0$.

Note that $w_{\mathrm{mo}}\left(T_{s} f-f\right)(t) \leq\left\|T_{s} f-f\right\|_{\mathrm{BMO}}$ for all $0<t \leq 1$.

On the other hand

$$
\begin{aligned}
w_{\mathrm{mo}}\left(T_{s} f-f\right)(t)= & \sup _{|I| \leq t} \frac{1}{|I|} \int_{I}\left|\left(T_{s} f-f\right)\left(e^{i \theta}\right)-m_{I}\left(T_{s} f-f\right)\right| \frac{d \theta}{2 \pi} \\
\leq & \sup _{|I| \leq t} \frac{1}{|I|} \int_{I}\left|T_{s} f\left(e^{i \theta}\right)-m_{I}\left(T_{s} f\right)\right| \frac{d \theta}{2 \pi} \\
& +\sup _{|I| \leq t} \frac{1}{|I|} \int_{I}\left|f\left(e^{i \theta}\right)-m_{I}(f)\right| \frac{d \theta}{2 \pi} \\
= & 2 w_{\mathrm{mo}}(f)(t)
\end{aligned}
$$

The Lebesgue dominated convergence theorem gives $\lim _{s \rightarrow 0^{+}}\left\|T_{s} f-f\right\|_{\mathrm{MO}^{p}}=0$.

\section{Integrable harmonic oscillation.}

Throughout this section, given $z \in \Delta \backslash\{0\}$, we denote by $I_{z}$ the open $\operatorname{arc}$ in $\mathbb{T}$ with midpoint $\frac{z}{|z|}$ and length $\left|I_{z}\right|=1-|z|$. Given an $\operatorname{arc} I \subset \mathbb{T}$ and $\lambda \leq|I|^{-1}$ we shall write $\lambda I$ for the arc with the same midpoint and length $\lambda|I|$.

Let us collect several known facts to be used later on. 
Lemma 3.1. There exist constants $0<C, C_{1}, C_{2}, C_{3}<\infty$ such that

(i) $1-|z| \leq\left|e^{i \theta}-z\right| \leq C(1-|z|), e^{i \theta} \in I_{z}$, and $z \in \Delta$.

(ii) $C_{1} \frac{1}{\left|I_{z}\right|} \leq P_{z}\left(e^{i \theta}\right) \leq C_{2} \frac{1}{\left|I_{z}\right|}, e^{i \theta} \in I_{z}$, and $z \in \Delta$.

(iii) $\frac{1}{4^{k}\left|I_{z}\right|} \leq P_{z}\left(e^{i \theta}\right) \leq C_{3} \frac{1}{4^{k}\left|I_{z}\right|}, e^{i \theta} \in 2^{k} I_{z} \backslash 2^{k-1} I_{z}, k \in\{1,2, \ldots, N+1\}$, where $N=\left[\log _{2} \frac{1}{\left|I_{z}\right|}\right]$ and $z \in \Delta$.

Proof. All the statements follow from the estimates

$$
1-|z| \leq\left|e^{i \theta}-z\right| \leq\left|e^{i \theta}-\frac{z}{|z|}\right|+(1-|z|)
$$

and

$$
\left|e^{i \theta}-\frac{z}{|z|}\right| \leq\left|e^{i \theta}-z\right|+(1-|z|)
$$

For $0<p<\infty$ we define

$$
\|f\|_{\mathrm{HO}^{p}}=\|f\|_{L^{1}(\mathbb{T})}+\left(\int_{0}^{1}\left[w_{\mathrm{ho}}(f)(t)\right]^{p} \frac{d t}{t}\right)^{1 / p}
$$

to get a quasi-norm in the space $\mathrm{HO}^{p}(\mathbb{T})$.

Proposition 3.2. If $f \in L^{1}(\mathbb{T})$ and $0<t \leq 1$ then $w_{\mathrm{mo}}(f)(t) \leq c w_{\mathrm{ho}}(f)(t)$.

Proof. Let $I \subseteq \mathbb{T}$ be an arc such that $|I| \leq t$. Consider $z \in \Delta$ for which $I=I_{z}$. From $\left|I_{z}\right|=1-|z| \leq t$ we have $1-t \leq|z|<1$.

Using (ii) in Lemma 3.1 we have

$$
\begin{aligned}
\frac{1}{|I|} \int_{I}\left|f\left(e^{i \theta}\right)-m_{I}(f)\right| \frac{d \theta}{2 \pi} \leq & \frac{1}{\left|I_{z}\right|} \int_{I_{z}}\left|f\left(e^{i \theta}\right)-P(f)(z)\right| \frac{d \theta}{2 \pi} \\
& +\left|m_{I}(f)-P(f)(z)\right| \\
\leq & \frac{2}{\left|I_{z}\right|} \int_{I_{z}}\left|f\left(e^{i \theta}\right)-P(f)(z)\right| \frac{d \theta}{2 \pi} \\
\leq & C\left(\int_{-\pi}^{\pi}\left|f\left(e^{i \theta}\right)-P(f)(z)\right| P_{z}(\theta) \frac{d \theta}{2 \pi}\right) \\
\leq & C w_{\mathrm{ho}}(f)(t)
\end{aligned}
$$

Now taking the supremum over all arcs we get $w_{\text {mo }}(f)(t) \leq C w_{\text {ho }}(f)(t)$.

Theorem 3.3. Let $0<p<\infty$. Then $\operatorname{HO}^{p}(\mathbb{T})=\mathrm{MO}^{p}(\mathbb{T})$ with equivalent quasinorms. 
Proof. $\mathrm{HO}^{p}(\mathbb{T}) \subseteq \mathrm{MO}^{p}(\mathbb{T})$ follows from Proposition 3.2.

Assume now that $f \in \mathrm{MO}^{p}(\mathbb{T})$. Let us show that $f \in \mathrm{HO}^{p}(\mathbb{T})$ and $\|f\|_{\mathrm{HO}^{p}} \leq$ $C\|f\|_{\mathrm{MO}^{p}}$.

Let $t \in(0,1]$. For any $z \in \Delta$ with $|z|=1-t$ we consider the $\operatorname{arc} I=I_{z}$, which gives $\left|I_{z}\right|=1-|z|=t$. Take $N$ and $I_{k}(k=0,1, \ldots, N)$ so that $I_{k}=2^{k} I_{z}$ where $I_{0}=I, I_{N-1} \subsetneq \mathbb{T}$, and $I_{N}=\mathbb{T}$.

Using (iii) in Lemma 3.1 we have

$$
\begin{aligned}
& \int_{\mathbb{T}}\left|f\left(e^{i \theta}\right)-P(f)(z)\right| P_{z}\left(e^{i \theta}\right) \frac{d \theta}{2 \pi} \\
& \leq 2 \int_{\mathbb{T}}\left|f\left(e^{i \theta}\right)-m_{I}(f)\right| P_{z}\left(e^{i \theta}\right) \frac{d \theta}{2 \pi} \\
& \leq C\left(\int_{I}\left|f\left(e^{i \theta}\right)-m_{I}(f)\right| P_{z}\left(e^{i \theta}\right) \frac{d \theta}{2 \pi}\right. \\
&\left.+\sum_{k=1}^{N} \int_{I_{k} \backslash I_{k-1}}\left|f\left(e^{i \theta}\right)-m_{I}(f)\right| P_{z}\left(e^{i \theta}\right) \frac{d \theta}{2 \pi}\right) \\
& \leq C\left(\frac{1}{|I|} \int_{I}\left|f\left(e^{i \theta}\right)-m_{I}(f)\right| \frac{d \theta}{2 \pi}\right. \\
&\left.+\sum_{k=1}^{N} \frac{1}{4^{k}|I|} \int_{I_{k} \backslash I_{k-1}}\left|f\left(e^{i \theta}\right)-m_{I}(f)\right| \frac{d \theta}{2 \pi}\right) \\
& \leq C\left(w_{\operatorname{mo}}(f)(t)+\sum_{k=1}^{N} \frac{1}{2^{k}\left|I_{k}\right|} \int_{I_{k}}\left|f\left(e^{i \theta}\right)-m_{I}(f)\right| \frac{d \theta}{2 \pi}\right) .
\end{aligned}
$$

On the other hand, by (4),

$$
\begin{aligned}
\frac{1}{\left|I_{k}\right|} \int_{I_{k}}\left|f\left(e^{i \theta}\right)-m_{I}(f)\right| \frac{d \theta}{2 \pi} \\
\leq \frac{1}{\left|I_{k}\right|} \int_{I_{k}}\left|f\left(e^{i \theta}\right)-m_{I_{k}}(f)+\left(\sum_{j=1}^{k} m_{I_{j}}(f)-m_{I_{j-1}}(f)\right)\right| \frac{d \theta}{2 \pi} \\
\leq \frac{1}{\left|I_{k}\right|} \int_{I_{k}}\left|f\left(e^{i \theta}\right)-m_{I_{k}}(f)\right| \frac{d \theta}{2 \pi}+\sum_{j=1}^{k}\left|m_{I_{j}}(f)-m_{I_{j-1}}(f)\right| \\
\leq \frac{1}{\left|I_{k}\right|} \int_{I_{k}}\left|f\left(e^{i \theta}\right)-m_{I_{k}}(f)\right| \frac{d \theta}{2 \pi}+\sum_{j=1}^{k} \frac{\left|I_{j}\right|}{\left|I_{j-1}\right|} w_{\mathrm{mo}}(f)\left(\left|I_{j}\right|\right) \\
\leq w_{\mathrm{mo}}(f)\left(\left|I_{k}\right|\right)+\sum_{j=1}^{k} 2 w_{\mathrm{mo}}(f)\left(\left|I_{j}\right|\right) \\
\leq(1+2 k) w_{\mathrm{mo}}(f)\left(\left|I_{k}\right|\right) .
\end{aligned}
$$


Combining both estimates one gets

$$
\int_{\mathbb{T}}\left|f\left(e^{i \theta}\right)-P(f)(z)\right| P_{z}\left(e^{i \theta}\right) \frac{d \theta}{2 \pi} \leq C\left(w_{\mathrm{mo}}(f)(t)+\sum_{k=1}^{N} \frac{1+2 k}{2^{k}} w_{\mathrm{mo}}(f)\left(\left|I_{k}\right|\right)\right) .
$$

Taking the supremum over $\{z:|z|=1-t\}$ and using $\left|I_{k}\right|=2^{k} t$ we obtain

$$
\sup _{|z|=1-t} \int_{\mathbb{T}}\left|f\left(e^{i \theta}\right)-P(f)(z)\right| P_{z}\left(e^{i \theta}\right) \frac{d \theta}{2 \pi} \leq C\left(w_{\mathrm{mo}}(f)(t)+\sum_{k=1}^{N_{t}} \frac{1+2 k}{2^{k}} w_{\mathrm{mo}}(f)\left(2^{k} t\right)\right)
$$

where $N=N_{t}=\left[\log _{2} \frac{1}{t}\right]+1$. This implies that

$$
w_{\mathrm{ho}}(f)(t) \leq C\left(w_{\mathrm{mo}}(f)(t)+\sum_{k=1}^{N_{t}} \frac{1+2 k}{2^{k}} w_{\mathrm{mo}}(f)\left(2^{k} t\right)\right) .
$$

For $0<p<1$ we have

$$
\left[w_{\mathrm{ho}}(f)(t)\right]^{p} \leq C_{p}\left(\left[w_{\mathrm{mo}}(t)(t)\right]^{p}+\sum_{k=1}^{N_{t}} \frac{(1+2 k)^{p}}{2^{p k}}\left[w_{\mathrm{mo}}(f)\left(2^{k} t\right)\right]^{p}\right) .
$$

For $p \geq 1$ we apply Hölder's inequality to obtain

$$
\left[w_{\mathrm{ho}}(f)(t)\right]^{p} \leq C_{p}\left(\left[w_{\mathrm{mo}}(t)(t)\right]^{p}+\sum_{k=1}^{N_{t}} \frac{(1+2 k)^{p}}{2^{k}}\left[w_{\mathrm{mo}}(f)\left(2^{k} t\right)\right]^{p}\right) .
$$

Now integrating, and taking into account that $1 \leq k \leq N_{t}=\left[\log _{2} \frac{1}{t}\right]+1$ is equivalent to $0<t \leq 2^{-k}$, we get

$$
\begin{aligned}
\int_{0}^{1}\left[w_{\mathrm{ho}}(f)(t)\right]^{p} \frac{d t}{t} & \leq C_{p} \int_{0}^{1}\left[w_{\mathrm{mo}}(t)(t)\right]^{p} \frac{d t}{t}+C_{p} \int_{0}^{1} \sum_{k=1}^{N_{t}+1} \frac{(1+2 k)^{p}}{2^{k \min \{p, 1\}}}\left[w_{\mathrm{mo}}(f)\left(2^{k} t\right)\right]^{p} \frac{d t}{t} \\
& \leq C_{p}\|f\|_{M O^{p}}^{p}+C_{p} \sum_{k=1}^{\infty} \frac{(1+2 k)^{p}}{2^{k \min \{p, 1\}}} \int_{0}^{2^{-k}}\left[w_{\mathrm{mo}}(f)\left(2^{k} t\right)\right]^{p} \frac{d t}{t} \\
& \leq C_{p}\|f\|_{M O^{p}}^{p}+C_{p} \sum_{k=1}^{\infty} \frac{(1+2 k)^{p}}{2^{k \min \{p, 1\}}} \int_{0}^{1}\left[w_{\mathrm{mo}}(f)(t)\right]^{p} \frac{d t}{t} \\
& \leq C\|f\|_{M O^{p}}^{p} .
\end{aligned}
$$

Putting together all the estimates we have the result. 


\section{References}

[1] A. Baernstein II, Analytic functions of bounded mean oscillation, Aspects of contemporary complex analysis (Proc. NATO Adv. Study Inst., Univ. Durham, Durham, 1979), 1980, pp. 3-36.

[2] S. Campanato, Proprietà di hölderianità di alcune classi di funzioni, Ann. Scuola Norm. Sup. Pisa (3) 17 (1963), 175-188.

[3] __ Proprietà di una famiglia di spazi funzionali, Ann. Scuola Norm. Sup. Pisa (3) 18 (1964), $137-160$.

[4] J. R. Dorronsoro, Mean oscillation and Besov spaces, Canad. Math. Bull. 28 (1985), no. 4, $474-480$.

[5] K. M. Dyakonov, Besov spaces and outer functions, Michigan Math. J. 45 (1998), no. 1, 143-157.

[6] C. Fefferman, Characterizations of bounded mean oscillation, Bull. Amer. Math. Soc. 77 (1971), $587-588$.

[7] J. B. Garnett, Bounded analytic functions, Pure and Applied Mathematics, vol. 96, Academic Press Inc. [Harcourt Brace Jovanovich Publishers], New York, 1981.

[8] D. Girela, Analytic functions of bounded mean oscillation, Complex function spaces (Mekrijärvi, 1999), 2001, pp. 61-170.

[9] H. Greenwald, On the theory of homogeneous Lipschitz spaces and Campanato spaces, Pacific J. Math. 106 (1983), no. 1, 87-93.

[10] S. Janson, Generalizations of Lipschitz spaces and an application to Hardy spaces and bounded mean oscillation, Duke Math. J. 47 (1980), no. 4, 959-982.

[11] F. John and L. Nirenberg, On functions of bounded mean oscillation, Comm. Pure Appl. Math. 14 (1961), 415-426.

[12] J. Peetre, On the theory of $\mathcal{L}_{p},{ }_{\lambda}$ spaces, J. Functional Analysis 4 (1969), 71-87.

[13] F. Ricci and M. Taibleson, Boundary values of harmonic functions in mixed norm spaces and their atomic structure, Ann. Scuola Norm. Sup. Pisa Cl. Sci. (4) 10 (1983), no. 1, 1-54.

[14] D. Sarason, Function theory on the unit circle, Virginia Polytechnic Institute and State University Department of Mathematics, Blacksburg, Va., 1978.

[15] W. S. Smith, $\operatorname{BMO}(\rho)$ and Carleson measures, Trans. Amer. Math. Soc. 287 (1985), no. 1, $107-126$.

[16] S. Spanne, Some function spaces defined using the mean oscillation over cubes, Ann. Scuola Norm. Sup. Pisa (3) 19 (1965), 593-608.

[17] B. E. Viviani, An atomic decomposition of the predual of $\operatorname{BMO}(\rho)$, Rev. Mat. Iberoamericana 3 (1987), no. 3-4, 401-425. 